\title{
Correction to: Association Between School Bullying, Suicidal Ideation, and Eating Disorders Among School-Aged Children from Antioquia, Colombia
}

\author{
Jessica Quintero Jurado ${ }^{1}$ Nadia Moratto Vásquez ${ }^{1}$. \\ Beatriz Caicedo-Velasquez ${ }^{2,3}$ (1) Natalia Cárdenas Zuluaga ${ }^{1}$. \\ Dorothy L. Espelage ${ }^{4}$
}

Published online: 4 October 2021

(c) Associação Brasileira de Psicologia 2021

\section{Correction to: Trends in Psychology https://doi.org/10.1007/s43076-021-00101-2}

This article was originally published with the wrong affiliation of the third author, Dr. Beatriz Caicedo-Velasquez, so it was updated to correct the author's affiliation: Incorrect author's affiliation:

Beatriz Caicedo-Velasquez

Grupo de Investigación en Psicología, Salud y Sociedad, Universidad CES, Medellín, Colombia

Facultad Nacional de Salud Pública, Universidad de Antioquia, Medellín, Colombia ORCID: https://orcid.org/0000-0001-6284-512X

The original article has been corrected

The original article can be found online at https://doi.org/10.1007/s43076-021-00101-2.

Jessica Quintero Jurado

quinteroj.jessica@uces.edu.co

1 Grupo de Investigación en Psicología, Salud y Sociedad, Universidad CES, Medellín, Colombia

2 Facultad Nacional de Salud Pública, Universidad de Antioquia, Medellín, Colombia

3 Grupo de Investigación Epidemiología y Bioestadística, Universidad CES, Medellín, Colombia

4 University of North Carolina At Chapel Hill, Chapel Hill, NC, USA 